\title{
Minimal Order Linear Functional Observers Design for Multicell Series Converter
}

\author{
Mariem Jday $^{1}$ \\ Laboratoire de Recherche \\ en Automatique, \\ Ecole Nationale d'Ingénieurs de Tunis, \\ Université de Tunis El Manar \\ Le Belvédère, 1002 Tunis, Tunisia
}

\author{
Paul-Etienne Vidal $^{2}$ \\ Laboratoire de Génie \\ de Production,
}

\author{
Joseph Haggège ${ }^{3}$ \\ Laboratoire de Recherche \\ en Automatique,
}

\begin{abstract}
The requirement of high voltage-power level for many applications like the energy conversion system give rise to use a structure of a multilevel converter like the multicell series converter. To benefit as much as possible from this power converter an appropriate voltage distribution for each cell must be performed, hence the need to estimate this voltages. This paper aims to design a minimal single linear functional observer for a multicell series converter to estimate the capacitor voltages. Based on its hybrid model, an observability study prove the ability to estimate this capacitor voltages. Also a linear functional observers are proposed using a direct procedure without solving the Sylvester equation and based on an operation mode classification approach. Simulations of four-cells multicell converter are given in order to check the efficiency of the converter's hybrid model and the performance of the proposed minimal single linear functional observers.
\end{abstract}

Keywords-Multicell converter; voltage capacitor; hybrid model; Z(TN)-Observability; functional observer

\section{INTRODUCTION}

Due to the energy transition combined with e-mobility, energy conversion systems require higher power level. This can be achieved with an increase of the functioning current or voltage. The increase of the functioning voltage is done in usual power structures with an increase of the switches's voltages. For instance, [1] illustrates how to apply $10 \mathrm{kV}$ Silicon switches ( $\mathrm{Si}$ ) in a two voltage level converter. However, high blocking voltage switches have a lower dynamic performance. To mitigate both power level associated with higher blocking voltage, and good switching dynamics, Multicell Series Converter (MSC) are an alternative to usual conversion structures. In such a structure, the output leg voltage is produced by a specific association of switching cells. The main advantage of MSC is to increase the degree of freedom of the conversion structure and then to contribute to the reduction of the voltage constraints on the switches, such as the reduce of the harmonic distortion rate which lead to obtain a high quality output voltage [2], [3]. Nevertheless, MSC structures have inner capacitors. Each capacitor voltages must have a specific value according to the applied input voltage. This is done according to a suitable control strategy applied. A risk known of such a structure is the capacitor voltage imbalance [3], which should be avoided. As a matter of facts, capacitor voltage must be known at each time. This is possible by the mean of sensorless strategy, also called observers, instead of the extra sensors which can increase complexities and costs of converters. Due to the converter structure complexity, one drawback of sensorless techniques is the observability statement [4]. Hence, it is needed to proceed to an observability study before the observer design. The aim is to prove the possibility to estimate the voltage capacitors. For MSC, the observer design can be made with two types of model: the average model and the instantaneous model. In the MSC average model, the non linear behavior of the system is highlighted, so a non linear observer must be used such as the non linear sliding observer presented in [5].

The second model is the instantaneous hybrid model which expresses the dynamic of continuous variable according to the state of discrete variables.

Several types of observers based on this model are developed, such as the adaptive linear observer given in [6], [7] and the sliding mode observer presented in [8].

In order to reduce the estimation algorithm complexity, linear observers can be an interesting alternative. In order to avoid non-linearities observers should be designed from the instantaneous model. Among this observers, the linear functional observer (LFO) which is a low order observer is can be used, it allows to estimate a function of the state vector. In particular each capacitor voltage of the MSC can be estimated thanks a state function.

Recently, [9] detailed the design of the functional observer for switched discrete system.

Furthermore, it is noticed that the study of the functional observer for a multilevel converter (even for a continue switch system) has not been reported despite its utility for such system which present an observability problem caused by a switch behaviour. This paper tackle this problem. An observability study and an appropriate strategy to estimate the MSC voltages are given for an hybrid model of the MSC. Thereafter an observation strategy using the functional observer is defined allowing to isolate each capacitor voltage and reduce the complexity of the observer design strategy. The procedure to get the minimal order functional observer presented in this paper is based on the work of F. Rotella et al. [10], in which, it is demonstrated that the solution of Sylvester equation is not given by using this direct method. This work provides the ability to set an arbitrary dynamics to this observer to increase its performance. 
The paper is arranged as follows. In Section 2 the hybrid model of the multi-cell series converter is given. Section 3 discusses the problem of the observability of the voltage capacitors. Section 4 is dedicated to the design of the functional observers for this system. Before the conclusion an application to a four-cells converter is provided including simulation results.

\section{Model of Multicell Series INVERTER}

The multicell serie converter [11] [12] [13] was introduced in the 90's . The inverter structure of a MSC is illustrated in Fig. 1. The MSC is a serial connection of $p$ pairs of switches

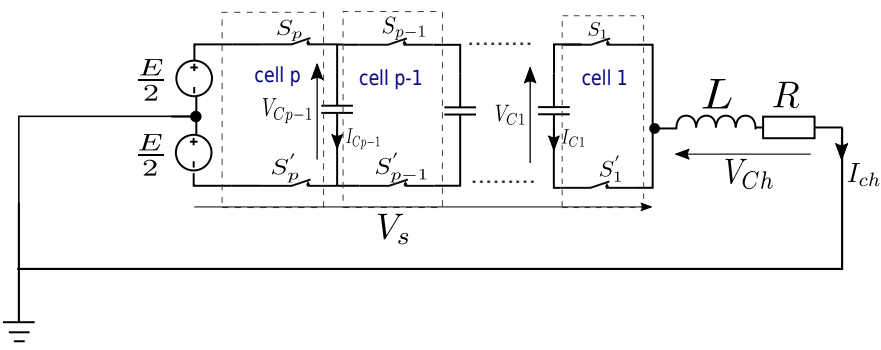

Fig. 1. Multi-cell series inverter.

$\left\{S_{j}, S_{j}^{\prime}\right\}$. The switches $S_{j}$ and $S_{j}^{\prime}$ are grouped into switching cells where each switch $S_{j}$ is associated to its binary state such as $S_{j} \in\{0,1\}, S_{j}=\bar{S}_{j}^{\prime} . j \in\{1, \ldots, p\}, j \in\{\mathbb{N}\}$.

$I_{C h}$ and $V_{C h}$ are respectively the load current and voltage , $E$ is the constant input voltage, $L$ and $R$ are respectively the inductance and resistance of the load and $V_{s}$ is the leg voltage. The inner voltage floating capacitors are $V_{C 1}, \ldots, V_{C p-1}$. As there is $p-1$ capacitors inserted, it exists $p-1$ capacitor voltages and currents such as:

$$
\begin{gathered}
V_{C j}=\frac{j E}{p}, \\
I_{C j}=I_{C h} \times\left(S_{(j+1)}-S_{j}\right),
\end{gathered}
$$

where $j$ ponderates the input voltage $E$.

The relationship between the leg voltage $V_{s}$ and the load voltage $V_{C h}$ is:

$$
V_{C h}=V_{s}-\frac{E}{2}=L \frac{d I_{C h}}{d t}+R I_{C h} .
$$

Then, the load current $I_{C h}$ is:

$$
\dot{I}_{C h}=\frac{V_{s}}{L}-\frac{R}{L} I_{C h}-\frac{E}{2 L} .
$$

As stated in [?], equations (3) and (4) allow to express the leg voltage $V_{s}$ as:

$$
V_{s}=E S_{p}+\sum_{j=1}^{p-1} V_{C j}\left(S_{j}-S_{(j+1)}\right) .
$$

Indeed, the new differential equation of the load current is defined by:

$$
\begin{aligned}
\dot{I}_{C h} & =-\frac{R}{L} I_{C h}+\frac{S_{p} E}{L}-\frac{E}{2 L} \\
& -\sum_{j=1}^{p-1} \frac{V_{C j}}{L}\left(S_{(j+1)}-S_{j}\right) .
\end{aligned}
$$

Subsequently, the dynamic behavior of the voltage capacitor $V_{C j}$ is expressed as follow:

$$
\dot{V}_{C j}=I_{C h} \frac{1}{C_{j}}\left(S_{(j+1)}-S_{j}\right) .
$$

$d_{j}=S_{(j+1)}-S_{j}$ and $d_{p}=S_{p}$ will be further introduced in order to simplify the expression. Let us remark that $m$ is the operating mode. It is a discrete state variable of the system and it depends of the input signal $d_{j}$ (and so $S_{j}$ ).

Let us consider the state vector $x=$ $\left[V_{C 1}, V_{C 2}, \ldots, V_{C p-1}, I_{C h}\right]^{T}$. As a matter of facts, the MSC instantaneous model is defined as a state space representation such as:

$$
\left\{\begin{array}{l}
\dot{x}(t)=A_{m} x(t)+B_{m} u(t) \\
y=C x(t)
\end{array}\right.
$$

where:

$$
\begin{aligned}
& A_{m}=\left[\begin{array}{cccc}
0 & \cdots & 0 & \frac{d_{1}}{c_{1}} \\
\vdots & \ddots & \vdots & \vdots \\
0 & \cdots & 0 & \frac{d_{(p-1)}}{c_{(p-1)}} \\
-\frac{d_{1}}{L} & \cdots & -\frac{d_{(p-1)}}{L} & -\frac{R_{C h}}{L_{C h}}
\end{array}\right] \\
& B_{m}=\left[\begin{array}{llll}
0 & \cdots & 0 & \frac{d_{p}}{L_{C h}}-\frac{1}{2 L_{C h}}
\end{array}\right]^{T}, \\
& C=\left[\begin{array}{llll}
0 & \cdots & 0 & 1
\end{array}\right] .
\end{aligned}
$$

In such a structure, $u(t)=E$, and $y(t)$ is the measure available in the MSC which corresponds to the load current.

$A_{m}(p \times p), B_{m}(p \times 1), C(1 \times p)$ are a variable matrices which depend on $m$.

The number of $m$ is defined according to the number of cells, such as $m=2^{p}$ for $p$ cells. Subsequently, it is noticed that this model merges continuous variables $\left(I, V_{C j}\right)$ and discrete variables $\left(S_{1}, S_{2}, . ., S_{p}\right)$. This is present an hybrid model of the multicell converter. To simulate the multi-cell converter with the hybrid approach every matrices $A_{m}$ and $B_{m}$ have to be computed. As stated in Fig. 2, the Pulse Width Modulation (PWM) provides the input order allowing to specify the operating mode $m$. According to $m$, the system switches to the appropriate matrices $A$ and $B$.

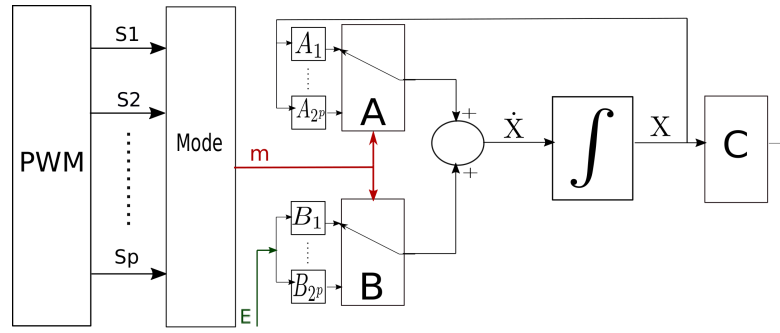

Fig. 2. Simulation model of the multicell series converter. 


\section{OBSERVABILITY ANALYSIS}

\section{A. Hybrid Observability}

Let us introduce the hybrid observability also known as the $Z\left(T_{N}\right)$-observability [14]. On the one hand, the hybrid time trajectory denoted $T_{N}$ is a finite or infinite interval sequence $T_{N}=\left\{I_{i}\right\}_{i=0, N}$ such as:

- $I_{i}=\left[t_{i, 0}, t_{i, 1}\right], I_{i}>0$ for all $0 \leq i \leq N$,

- For all $0 \leq i \leq N, t_{i, 1}=t_{i+1,0}$,

- $t_{0,0}=t_{\text {init }}$ and $t_{N, 1}=t_{\text {end }}$.

On the other hand, $\left\langle T_{N}\right\rangle$ is the ordered list of the discrete input $d_{j}$ associated to the trajectory $T_{N}$. It orders the operating mode applied during the interval $I_{i}$ such as $\left\langle T_{N}\right\rangle=\left\{\ldots m_{i} \ldots\right\}$.

Let us define the vector $Z(t, x)$ and the projection $P_{i}$ such as $P_{i} Z(t, x)$, is a state function of $Z(t, x)$ containing one state variable.

The $Z\left(T_{N}\right)$-observability consider the system (8) and a fixed hybrid time trajectory [14] $T_{N}$ and $\left\langle T_{N}\right\rangle$. Suppose that $z=Z(t, x)$ is always continuous under any admissible control input. If there is a sequence of linear projection $\left\{P_{i}\right\}_{i=0, N}$ such as:

-for any $0 \leq i \leq N, P_{i} Z(t, x)$ is Z-observable $t \in I_{i}$, - $\operatorname{rank}\left(\left[P_{0}^{T}, \cdots, P_{N}^{T}\right]\right)=\operatorname{dim}(\mathrm{Z})=\mathrm{n}_{\mathrm{z}}$,

- $\frac{d \bar{P}_{i} Z(t, x)}{d t}=0$ for $t \in I_{i}$ where $\left\{\bar{P}_{i}^{T}, P_{i}^{T}\right\}$ has a full rank in $\mathbb{R}^{\operatorname{dim}(\mathrm{z}) \times \operatorname{dim}(\mathrm{z})}$, then $z$ is $Z$-observable with respect to the hybrid time trajectory $T_{N}$ and $\left\langle T_{N}\right\rangle$.

The first condition indicates that a projection of the state variable $P_{i} Z(t, x)$ is observable, at least, on a time interval $I_{i}$ of an hybrid time trajectory $T_{N}$. The second implies that all the state variables must be observable for $T_{N}$ including $I_{i}$. The third condition ensures that the inobservable variable must remain constant during $I_{i}$.

\section{B. Application of the Hybrid Observability to the MSC}

Let us consider the converter's model (8), the observable vector $Z(t, x)=\left[V_{C 1}, V_{C 2}, \ldots, V_{C p-1}\right]^{T}$ and the projection $P_{i}$ such as:

$$
P_{i} Z(t, x)=\left[\begin{array}{cccc}
\delta_{1} & 0 & \cdots & 0 \\
0 & \delta_{2} & \ddots & \vdots \\
\vdots & \ddots & \ddots & 0 \\
0 & \cdots & 0 & \delta_{p-1}
\end{array}\right]\left[\begin{array}{c}
V_{C 1} \\
V_{C 2} \\
\vdots \\
V_{C p-1}
\end{array}\right],
$$

where for $k=\{1, \ldots, p-1\}, \delta_{k} \in\{0,1\}$. It is noticed that our wish is to observe one capacitor voltage for an interval $I_{i}$, then a single $\delta_{k}$ must be equal to 1 .

As exemplified by [15], [16] and [17], the application of the hybrid observability yields to the following results.

The PWM strategy generates the control order $d_{j}, j \in$ $\{1, \ldots, p\}$ such as each order is associated to an operating mode $m_{i}$ during a time interval $I_{i}, i \in\{1, \ldots, N\} . T_{N}=\left\{I_{i}\right\}$ is the hybrid time trajectory and $\left\langle T_{N}\right\rangle=\left\{\ldots m_{i} \ldots\right\}$.
Using the following equation:

$$
\dot{I}_{C h}=-\frac{R}{L} I_{C h}+\frac{d_{p} E}{L}-\frac{E}{2 L}-\frac{V_{C 1}}{L} d_{1}-\ldots-\frac{V_{C p-1}}{L} d_{p-1},
$$

it is noticed that the derivatives of the measured current allows to obtain $p-1$ independent equations that used to compute the projection $P_{j}$, such as $V_{C j}=P_{j} Z(t, x)$ obtained from the measurement of $I_{c h}$.

Consequently $\operatorname{rank}\left(\left[\mathrm{P}_{1}^{\mathrm{T}}, \mathrm{P}_{2}^{\mathrm{T}}, \ldots, \mathrm{P}_{\mathrm{N}}^{\mathrm{T}}\right]\right)=p-1$.

Effectively in such control sequences $d_{j}$, the unobservable voltages are constants $\left(\dot{V}_{C j}=0\right)$, this is verified using equation (14).

$$
\dot{V}_{C j}=I_{C h} \frac{1}{C_{j}} d_{j} .
$$

Consequently, the MSC is observable with respect to the hybrid time trajectory $T_{N}$ and the ordered list $\left\langle T_{N}\right\rangle$.

\section{OBSERVER DESIGN}

\section{A. Observation Problem Statement}

According to the previous observability study, each voltage $V_{C j}, j=\{1, \ldots, p-1\}$ is observable according to the current measurement. As the observability is related to the mode $m$, the capacitor voltages are not observable through the same mode $m$, so it is not possible to apply a full rank observer on the hybrid model (8). The solution proposed by [18] is to break down the model into non linear sub-models where each one describes the dynamic of one voltage capacitor. Then a full rank observers were applied to the sub-models. Using this proposed solution the complexity of the estimation algorithms was increased. The purpose of our study is to develop a minimal order linear functional observers for the MSC's hybrid model in order to estimate each capacitor voltage. In fact each linear functional observer allows to estimate a linear state function of a given voltage capacitor. This section deals with the direct strategy to design a minimal order functional observer to estimate one voltage capacitor from the measurement of the load current and the knowledge of the control input.

\section{B. Functional Observer Definition}

The functional observer [19] [20] [21] [22] is a low order observer used to estimate a linear function of the state :

$$
v(t)=P x(t),
$$

where $P$ is $\mathbb{R}^{(f \times n)}$ matrix, $n$ is the order of the system and $f$ is the number of rows of $P$. For a linear dynamic system, the linear functional observer is expressed as follow:

$$
\left\{\begin{array}{r}
\dot{z}(t)=F z(t)+G u(t)+H y(t) \\
\omega(t)=L z(t)+V y(t) .
\end{array}\right.
$$

where $F, G, H, L$ and $V$ are a constant matrices.

Let us define $q$ as the observer order and $e$ is the number of measures.

The objective of the study is to provide a minimal single linear observer to estimate the voltage capacitors of the MSC. On the one hand the word minimal indicates that the functional observer must have an order lower than $n-e-f$. Effectively, it 
is possible to distinguish several types of functional observers [22] according to their order. In the other hand the word single implies that $f=1$

Moreover, in [10], a direct procedure to design this observer is presented. It is demonstrated how to guarantee a minimal $q$-order observer. As an hybrid system modeled in (8) is considered, an hybrid observer is proposed as:

$$
\left\{\begin{array}{c}
\dot{z}_{i}(t)=F_{m, i} z_{m, i}(t)+G_{m, i} u(t)+H_{m, i} y(t) \\
w_{i}(t)=L_{m, i} z_{m, i}(t)+V_{m, i} y(t),
\end{array}\right.
$$

where: $m$ is the discrete state, $i$ is the linear function index, $i \in\{1, \ldots, s\}, s$ is the number of linear functional to observe and $n$ is the order of the system. This observer is developed to estimate the linear function $v_{i}(t)$ such as:

$$
v_{i}(t)=P_{i} x(t), P_{i} \in \mathbb{R}^{(1 \times n)}
$$

$F_{m, i}, G_{m, i}, H_{m, i}, L_{m, i}$ and $V_{m, i}$ are a variable matrices which depend on the operation mode $m$ and the linear projection $P_{i}$.

The following assumptions [23] [19] [20] have to be verified to ensure that a functional observer exists:

$$
\begin{gathered}
G_{m, i}=T_{m, i} B_{m}, \\
T_{m, i} A_{m}-F_{m, i} T_{m, i}=H_{m, i} C_{m}, \\
P_{i}=L_{m, i} T_{m, i}+V_{m, i} C_{m},
\end{gathered}
$$

where $T_{m, i}$ is defined as $\lim _{t \rightarrow \infty}\left(z_{i}(t)-T_{m, i} x(t)\right)=0$ where $e=z_{i}(t)-T_{m, i} x(t)$. Using (17), (19) and (20), the dynamic observation error is finally expressed as follow :

$$
\dot{e}_{m, i}(t)=F_{m, i} e_{m, i}(t)
$$

The stability of the observer is ensured by the asymptotic convergence of its error. Consquently, $F_{m, i}$ must be Hurwitz matrices.

The existence condition of a minimal order observer for a single linear functional where $q$ is the minimal order is such as:

$$
\operatorname{rank}\left(\Sigma_{\mathrm{q}, \mathrm{m}, \mathrm{i}}\right)=\operatorname{rank}\left(\left[\begin{array}{c}
\Sigma_{q, m, i} \\
P_{i} A_{m}^{q}
\end{array}\right]\right)
$$

where:

$$
\Sigma_{q, m, i}=\left[\begin{array}{c}
C_{m} \\
P_{i} \\
C_{m} A_{m} \\
P_{i} A_{m} \\
\vdots \\
C_{m} A_{m}^{q-1} \\
P_{i} A_{m}^{q-1} \\
C_{m} A_{m}^{q}
\end{array}\right] .
$$

Consequently, $P_{i} A_{m}^{q}$ must be linearly dependent of the columns $C_{m} A_{m}^{j}$ and $P_{i} A^{j}, j \in\{0, \ldots, q\}$. So it exists $\Gamma_{j, m, i}$ and $\Lambda_{j, m, i}$ for $j \in\{0, \ldots, q-1\}$ such as:

$$
P_{i} A_{m}^{q}=\sum_{j=0}^{q} \Gamma_{j, m, i} C_{m} A_{m}^{j}+\sum_{j=0}^{q-1} \Lambda_{j, m, i} P_{i} A_{m}^{j} .
$$

Let us consider the linear functional $v_{i}$ and its $q$-derivative:

$$
v_{i}^{(q)}(t)=P_{i} A_{m}^{q} x(t)+\sum_{j=0}^{q-1} P_{i} A_{m}^{q-1-j} B_{m} u^{(j)}(t) .
$$

Using the expression $(25), v^{(q)}(t)$ can be written as follows:

$$
\begin{array}{r}
v_{i}^{(q)}(t)=\sum_{j=0}^{q} \Gamma_{j, m, i} C_{m} A_{m}^{j} x(t)+\sum_{j=0}^{q-1} \Lambda_{j, m, i} P_{i} A_{m}^{j} x(t)+ \\
\sum_{j=0}^{q-1} P_{i} A_{m}^{j} B_{m} u^{(q-1-j)}(t) .
\end{array}
$$

In order to remove $x(t)$ from the last expression, it's possible to express $v_{i}^{(q)}(t)$ as follow:

$$
v_{i}^{(q)}(t)=\sum_{j=0}^{q} \Gamma_{j, m, i} y^{(i)}+\sum_{j=0}^{q-1} \Lambda_{j, m, i} v^{(j)}+\sum_{j=0}^{q-1} \phi_{j, m, i} u^{(j)}(t),
$$

where $k \in\{0, \ldots, q-2\}$ and $\Phi_{k}$ is expressed as follow:

$$
\begin{aligned}
\phi_{k}=\left[P_{i} A_{m}^{q-1-k}\right. & \left.-\sum_{j=k+1}^{q} \Gamma_{j, m, i} C_{m} A_{m}^{j-k-1}\right] B_{m} \\
& -\left[\sum_{j=k+1}^{q-1} \Lambda_{j, m, i} P_{i} A_{m}^{j-k-1}\right] B_{m},
\end{aligned}
$$

and

$$
\phi_{q-1}=\left[P_{i}-\Gamma_{q, m, i} C_{m}\right] B_{m} .
$$
(30).

Thus the observer matrices are presented in expression

$$
\begin{aligned}
& \dot{z}_{m, i}(t)= {\left[\begin{array}{cccc}
0 & & 0 & \Lambda_{0, m, i} \\
1 & \ddots & & \Lambda_{1, m, i} \\
0 & \ddots & 0 & \vdots \\
& & 1 & \Lambda_{q-1, m, i}
\end{array}\right] z(t)+\left[\begin{array}{c}
\phi_{0, m, i} \\
\phi_{1, m, i} \\
\vdots \\
\phi_{q-1, m, i}
\end{array}\right] u(t) } \\
&+ {\left[\begin{array}{c}
\Gamma_{0, m, i}+\Lambda_{0, m, i} \Gamma_{q, m, i} \\
\Gamma_{1, m, i}+\Lambda_{1, m, i} \Gamma_{q, m, i} \\
\vdots \\
\Gamma_{q-1, m, i}+\Lambda_{q-1, m, i} \Gamma_{q, m, i}
\end{array}\right] y(t) } \\
& \omega_{m, i}(t)=\left[\begin{array}{llll}
0 & \ldots & 0 & 1
\end{array}\right] z_{m, i}(t)+\Gamma_{q, m, i} y(t) .
\end{aligned}
$$

Note that a rigorous proof of this results for a dynamic system is established in [10]. According to this result it is possible to deduce the previous expression of an hybrid functional observer applied for each operation mode of the MSC. The parameters $\Gamma_{j, m, i}$ and $\Lambda_{j, m, i}$ used to define the observer matrices $F_{m, i}, G_{m, i}, H_{m, i}, L_{m, i}$ and $V_{m, i}$ are deduced. Finally, from expression (25), the observer gains 
can be obtained as follow:

$$
\begin{aligned}
& P_{i} A_{m} \Sigma_{q, m, i}^{\dagger}= \\
& {\left[\begin{array}{llllll}
\Gamma_{0, m, i} & \Lambda_{0, m, i} & \Gamma_{1, m, i} & \ldots & \Lambda_{q-1, m, i} & \Gamma_{q, m, i}
\end{array}\right],}
\end{aligned}
$$

where $\Sigma_{q, m, i}^{\dagger}$ is the pseudo inverse of $\Sigma_{q, m, i}$.

\section{Z(TN) Observability Applied to the MSC Hybrid Model}

The second condition of the $Z(T N)$-observability of the MSC states that: $\operatorname{rank}\left(\left[\left|\mathrm{P}_{1}\right|,\left|\mathrm{P}_{2}\right|, \ldots,\left|\mathrm{P}_{\mathrm{N}}\right|\right]\right)=\mathrm{p}-1$ such as if $P_{i} Z(t, x)$ is observable then, using expression (12), $\left|\delta_{i}\right|=1$ and for $k \neq i,\left|\delta_{k}\right|=0$.

To fulfill this condition the PWM control should be able to generate an order control which allow to have the elementary sequences $(1,0,0, \ldots, 0), \ldots,(0,0,0, \ldots, 1)$, hence the idea of using the following linear functions:

$$
\begin{aligned}
P_{1}=\left[\begin{array}{lllll}
1 & 0 & \ldots & 0 & 0
\end{array}\right], P_{2} & =\left[\begin{array}{lllll}
0 & 1 & 0 & \ldots & 0
\end{array}\right], \\
\ldots, P_{p-1} & =\left[\begin{array}{lllll}
0 & \ldots & 0 & 1 & 0
\end{array}\right] .
\end{aligned}
$$

To conclude, it means that $Z\left(T_{N}\right)$ observability allows stating that $P_{p-1}$ projection matrices exists for $V_{C p-1}$ capacitor voltage observers. The design of minimal linear functional observers applied to hybrid system leads that for every $P_{p-1}$ and for every operating mode it exists an observer as stated in (17) that can be expressed. Finally, as the $Z\left(T_{N}\right)$-observability is demonstrated for the MSC hybrid system in Section III-B, a battery of observer designed from the operating modes and per capacitor voltage, will be used for the hybrid trajectory $\left\{T_{N},<T_{N}>\right\}$.

The simulation model of the functional observer for MSC is shown in Fig. 3. The next section is dedicated the design of a minimal functional observer for a four cells MSC.

\section{EXAMPle OF FouR-CElls MSC}

Let us consider a four-cells MSC presented in Fig. 4. Sixteen possible modes of operation can be found.

Table I summarizes the characteristics of each operation mode of four-cells MSC. The level of the output voltage reached is also indicated. $d j=S_{(j+1)}-S-j$ and $d_{p}=S_{p}$ are defined knowing at each mode the switch state $S_{j}$.

It is noticed that $x(t)=\left[V_{C 1}, V_{C 2}, V_{C 3}, I_{c h}\right]^{T} \in \mathbb{R}^{4}$ is the state vector. Then, the hybrid model of the four-cells MSC is presented as follow:

$$
\begin{gathered}
A_{m}=\left[\begin{array}{cccc}
0 & 0 & 0 & \frac{d_{1}}{C} \\
0 & 0 & 0 & \frac{d_{2}}{C} \\
0 & 0 & 0 & \frac{d_{3}}{C} \\
-\frac{d_{1}}{L_{c h}} & -\frac{d_{2}}{L_{c h}} & -\frac{d_{3}}{L_{c h}} & -\frac{R_{C h}}{L_{C h}}
\end{array}\right], \\
B_{m}=\left[\begin{array}{ccccc}
0 & 0 & 0 & \frac{d_{p}}{L_{C h}}-\frac{1}{2 L_{C h}}
\end{array}\right], \\
C_{m}=\left[\begin{array}{llll}
0 & 0 & 0 & 1
\end{array}\right] .
\end{gathered}
$$

In order to check the accuracy of the proposed model, some simulations are done. The parameters used are given in Table II.



Fig. 3. Simulation model of the functional observer for MSC

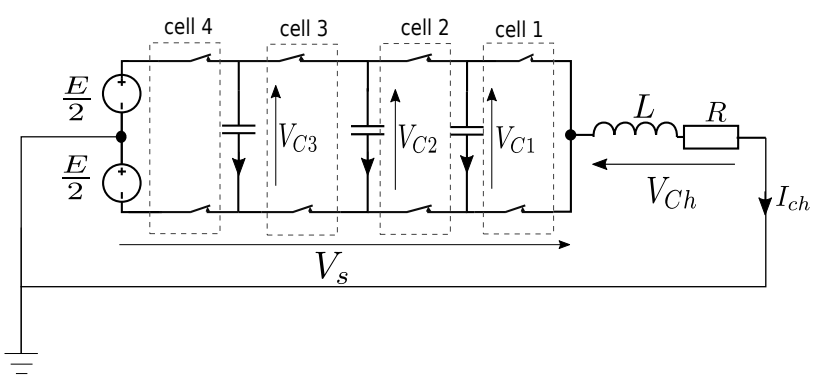

Fig. 4. Four-cells multicell series converter

TABLE I. OPERATIONS MODES FOR $\mathrm{P}=4$

\begin{tabular}{|c||c|c|c|c|c|}
\hline Mode $(m)$ & $d_{4}$ & $d_{3}$ & $d_{2}$ & $d_{1}$ & $V_{s}$ \\
\hline \hline 1 & 0 & 0 & 0 & 0 & 0 \\
\hline 2 & 0 & 0 & 0 & -1 & $V_{s}=V_{C 1}$ \\
\hline 3 & 0 & 0 & -1 & 1 & $V_{s}=V_{C 2}-V_{C 1}$ \\
\hline 4 & 0 & 0 & -1 & 0 & $V_{s}=V_{C 2}$ \\
\hline 5 & 0 & -1 & 1 & 0 & $V_{s}=V_{C 3}-V_{C 2}$ \\
\hline 6 & 0 & -1 & 1 & -1 & $V_{s}=V_{C 3}-V_{C 2}+V_{C 1}$ \\
\hline 7 & 0 & -1 & 0 & 1 & $V_{s}=V_{C 3}-V_{C 1}$ \\
\hline 8 & 0 & -1 & 0 & 0 & $V_{s}=V_{C 3}$ \\
\hline 9 & 1 & 1 & 0 & 0 & $V_{s}=E-V_{C 3}$ \\
\hline 10 & 1 & 1 & 0 & -1 & $V_{s}=E-V_{C 3}+V_{C 1}$ \\
\hline 11 & 1 & 1 & -1 & 1 & $V_{s}=E-V_{C 3}+V_{C 2}-V_{C 1}$ \\
\hline 12 & 1 & 1 & -1 & 0 & $V_{s}=E-V_{C 3}+V_{C 2}$ \\
\hline 13 & 1 & 0 & 1 & 0 & $V_{s}=E-V_{C 2}$ \\
\hline 14 & 1 & 0 & 1 & -1 & $V_{s}=E-V_{C 2}+V_{C 1}$ \\
\hline 15 & 1 & 0 & 0 & 1 & $V_{s}=E-V_{C 1}$ \\
\hline 16 & 1 & 0 & 0 & 0 & $E$ \\
\hline
\end{tabular}

TABLE II. SIMULATION PARAMETERS

\begin{tabular}{|c|c|c|c|c|c|}
\hline$E$ & $f_{P W M}$ & $f_{\text {modulante }}$ & $L_{c h}$ & $R$ & \multicolumn{2}{|c|}{$C_{1}, C_{2}, C_{3}$} \\
\hline $230 \mathrm{~V}$ & $1 \mathrm{kHz}$ & $50 \mathrm{~Hz}$ & $1 \mathrm{mH}$ & $10 \Omega$ & $4 \times 10^{-4} \mathrm{~F}$ \\
\hline
\end{tabular}


The simulation results allows to get the load voltage and the load current which are presented, respectively in Fig. 5 and Fig. 6. A sinus PWM is used with triangle carrier based signals.



Fig. 5. Load voltage

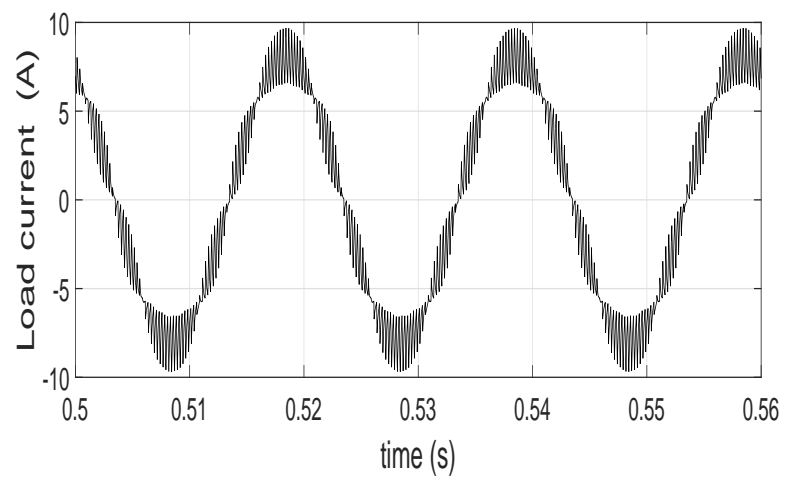

Fig. 6. Load current

In this control strategy, there are four triangular carrier waves with a phase shift of $\frac{2 \pi}{p}=\frac{\pi}{2}$ for each switching cell. The control strategy and the operating mode value are depicted in Fig. 7 and 8 for a one period $\frac{1}{f}=0.02 s$.

Fig. 7 and 8 shows that for a modulating time period, we pass through all the operating mode $m$.

\section{A. Design of Minimal Functional Observer}

The following steps illustrate the procedure to get the minimal functional observers in order to estimate $V_{C 1}$. In this case $i=1$, so:

$$
P_{1}=\left[\begin{array}{llll}
1 & 0 & 0 & 0
\end{array}\right] \text {. }
$$

It's noticed that this procedure is the same that can be used to estimate the other voltages of the MSC.

\section{1) Mode classification estimation approach:}

Our proposed observation approach is based on the $Z(T N)$ observability criterion. In fact, the $Z(T N)$-observability analysis prove the existence of a time interval $I_{i}$ associated to an operating mode through it the voltage $V_{C 1}$ is observable. During this interval the other voltages $\left(V_{C 2}\right.$ and $\left.V_{C 3}\right)$ are unobservable and keep constant. Also, it exist an operating

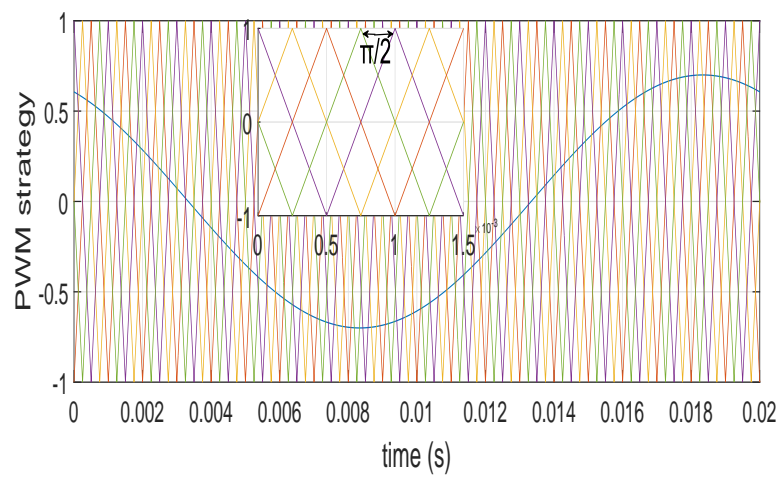

Fig. 7. PWM strategy

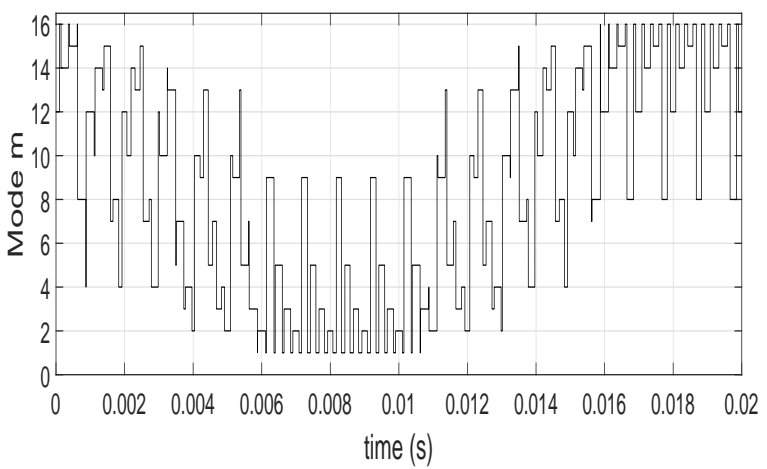

Fig. 8. Switching signal

modes in which $V_{C 1}$ is unobservable and constant. For other modes $V_{C 1}$ is not able to be estimated but it evolves over time, in this case there an estimation of a sum of voltage $V_{C j}$ which caused a $V_{C 1}$ voltage information gathering. Our proposed observation approach is based on this mode classification. Therefore, the first functional observers are designed for the operating modes that allows the estimation of $V_{C 1}$ ( modes 2 and 15). The second observers are designed for the modes in which $V_{C 1}$ is unobservable but evolves over time (modes 3, 6, 7, 10,11, 14). Finally, for the last class of modes, we set zero dynamics to the estimated voltage $\left(\dot{V}_{C 1}=0\right)$. This observation approach is presented in Fig. 9. The first step is to define the minimal order $q$ by using the



Fig. 9. Observation approach based on mode classification 
existence condition (23).

2) Operation modes 2 and 15 (Observable modes of $V_{C 1}$ ): Let us consider the observable modes 2 and 15 of $V_{C 1}$, for $q=1$ :

$$
\begin{aligned}
& \Sigma_{1,2,1}=\left[\begin{array}{c}
C \\
P_{1} \\
C A_{2}
\end{array}\right]=\left[\begin{array}{cccc}
0 & 0 & 0 & 1 \\
1 & 0 & 0 & 0 \\
1000 & 0 & 0 & -10000
\end{array}\right], \\
& \Sigma_{1,15,1}=\left[\begin{array}{c}
C \\
P_{1} \\
C A_{15}
\end{array}\right]=\left[\begin{array}{cccc}
0 & 0 & 0 & 1 \\
1 & 0 & 0 & 0 \\
-1000 & 0 & 0 & -10000
\end{array}\right],
\end{aligned}
$$

As $P_{1} A_{2}=\left[\begin{array}{llll}0 & 0 & 0 & -2500\end{array}\right]$ and $P_{1} A_{15}=$ $\left[\begin{array}{llll}0 & 0 & 0 & 2500\end{array}\right]$,

then

$$
\operatorname{rank}\left(\Sigma_{1,2,1}\right)=\operatorname{rank}\left(\left[\begin{array}{c}
\Sigma_{1,2,1} \\
P_{1} A_{2}
\end{array}\right]\right)=2
$$

and

$$
\operatorname{rank}\left(\Sigma_{1,15,1}\right)=\operatorname{rank}\left(\left[\begin{array}{c}
\Sigma_{1,15,1} \\
P_{1} A_{15}
\end{array}\right]\right)=2
$$

Consequently, a minimum observer can be designed to estimate the voltage $V_{C 1}$ of the four-cells MSC.

As $\Sigma_{1, m, 1}$ is singular and $\operatorname{dim}\left(\operatorname{ker}\left(\Sigma_{1, m, 1}\right)\right)=1$, for $m=$ $\{2,15\}$, the observer gains can be expressed as follow [24]:

$$
\begin{aligned}
& {\left[\begin{array}{lll}
\Gamma_{0, m, 1} & \Lambda_{0, m, 1} & \Gamma_{1, m, 1}
\end{array}\right]=P_{1} A_{m} \Sigma_{1, m, 1}^{\dagger}+} \\
& Z\left(I_{3}-\Sigma_{1, m, 1}^{\dagger} \Sigma_{1, m, 1}\right),
\end{aligned}
$$

where $\Sigma_{1, m, 1}^{\dagger}$ is the pseudo inverse matrix of $\Sigma_{1, m, 1}$ and $I_{3}$ is the $(3 \times 3)$ identity matrix and $Z$ an arbitrary matrix.

Let us consider the scalar $\lambda_{m}=Z \phi$ is the freedom degree such as $\phi X$ is the full rank factorization of $\left(I_{3}-\Sigma_{1, m, 1}^{\dagger} \Sigma_{1, m, 1}\right)$, so the gains observer are expressed as follow:

$$
\left[\begin{array}{lll}
\Gamma_{0, m, 1} & \Lambda_{0, m, 1} & \Gamma_{1, m, 1}
\end{array}\right]=P_{1} A_{m} \Sigma_{1, m, 1}^{\dagger}+\lambda_{m} X .
$$

For $m=\{2,15\}, \lambda_{m}$ is set such that $F_{m, 1}$ is a Hurwitz matrix. For this modes, the freedom degree allows to fix the observer dynamic. For $m=2$ the gains observer are expressed as follow:

$$
\begin{array}{r}
\Gamma_{0,2,1}=24.7525-0.995 \lambda_{2}, \\
\Lambda_{0,2,1}=-247.5248+0.0995 \lambda_{2}, \\
\Gamma_{1,2,1}=-0.2475-0.0001 \lambda_{2} .
\end{array}
$$

Based on expressions (30) and (42), $F_{2,1}$ is a Hurwitz matrix if $\lambda_{2}<2487.686$.

For $m=15$, the gains observer are expressed as follow:

$$
\begin{array}{r}
\Gamma_{0,15,1}=24.7525-0.995 \lambda_{15}, \\
\Lambda_{0,15,1}=-247.5248-0.0995 \lambda_{15}, \\
\Gamma_{1,15,1}=-0.2475-0.0001 \lambda_{15} .
\end{array}
$$

So, $F_{15,1}$ is a Hurwitz matrix if $\lambda_{15}>-2487.686$.
Based on observer matrices expressions (30), we obtain the observer gains for mode 2 and 15 which are illustrated in Table III. The obtained pole is -98.2 which indicate the stability of

TABLE III. OBSERVER GAINS FOR MODES 2 AND 15

\begin{tabular}{|c|c|c|c|c|c|c|}
\hline $\mathrm{m}$ & $\lambda_{m}$ & $F_{m, 1}$ & $G_{m, 1}$ & $H_{m, 1}$ & $L_{m, 1}$ & $V_{m, 1}$ \\
\hline 2 & 1500 & -98.2 & 49.13 & -1527 & 1 & 0.09 \\
\hline 15 & -1500 & -98.2 & 49.13 & 1527 & 1 & -0.09 \\
\hline
\end{tabular}

the minimal functional observers.

3) Case of modes 3, 6, 7, 10, 11 and 14 : Let us consider the modes $3,6,7,10,11$ and 14 in which $V_{C 1}$ is unobservable but it is not constant. We test the existence condition for $q=1$ and for the mode $m=3$.

$$
\Sigma_{1,3,1}=\left[\begin{array}{c}
C \\
P_{1} \\
C A_{3}
\end{array}\right]=\left[\begin{array}{cccc}
0 & 0 & 0 & 1 \\
1 & 0 & 0 & 0 \\
-1000 & 1000 & 0 & -10000
\end{array}\right] .
$$

As

$$
P_{1} A_{3}=\left[\begin{array}{llll}
0 & 0 & 0 & 2500
\end{array}\right],
$$

then

$$
\operatorname{rank}\left(\Sigma_{1,3,1}\right)=\operatorname{rank}\left(\left[\begin{array}{c}
\Sigma_{1,3,1} \\
P_{1} A_{3}
\end{array}\right]\right)=3 .
$$

This existence condition is also true for the operating modes $6,7,10,11,14$. Consequently, a minimum observer can be designed for this operation modes of the MSC.

As $\operatorname{dim}\left(\operatorname{ker}\left(\Sigma_{1, m, 1}\right)\right)=0$, for $m=3,6,7,10,11,14$, there is no freedom degree, so the observer gains are expressed as follow:

$$
\left[\begin{array}{lll}
\Gamma_{0, m, 1} & \Lambda_{0, m, 1} & \Gamma_{1, m, 1}
\end{array}\right]=P_{1} A_{m} \Sigma_{1, m, 1}^{\dagger} .
$$

Using the gains expression from (46), and based on a the expression (30) of the functional observer, the obtained gains observer values for this modes are presented in Table IV. From

TABLE IV. OBSERVER GAINS FOR MODES $3,6,7,10,11,14$

\begin{tabular}{|c|c|c|c|c|c|}
\hline $\mathrm{m}$ & $F_{m, 1}$ & $G_{m, 1}$ & $H_{m, 1}$ & $L_{m, 1}$ & $V_{m, 1}$ \\
\hline 3 & 0 & 0 & 2500 & 1 & 0 \\
\hline 6 & 0 & 0 & -2500 & 1 & 0 \\
\hline 7 & 0 & 0 & 2500 & 1 & 0 \\
\hline 10 & 0 & 0 & -2500 & 1 & 0 \\
\hline 11 & 0 & 0 & 2500 & 1 & 0 \\
\hline 14 & 0 & 0 & -2500 & 1 & 0 \\
\hline
\end{tabular}

Table III and IV we deduce that these operation modes are not intervene on observation dynamic of the voltage $V_{C 1}$. In fact, the observation dynamics and the convergence are defined through the modes 2 and 15 in which the output Ich depend only on the voltage $V_{C 1}$ thanks to the freedom degree. The same observer design strategy are used for the estimation of the voltage $V_{C 2}$ and $V_{C 3}$. The obtained gains observer values are given in the appendix. 


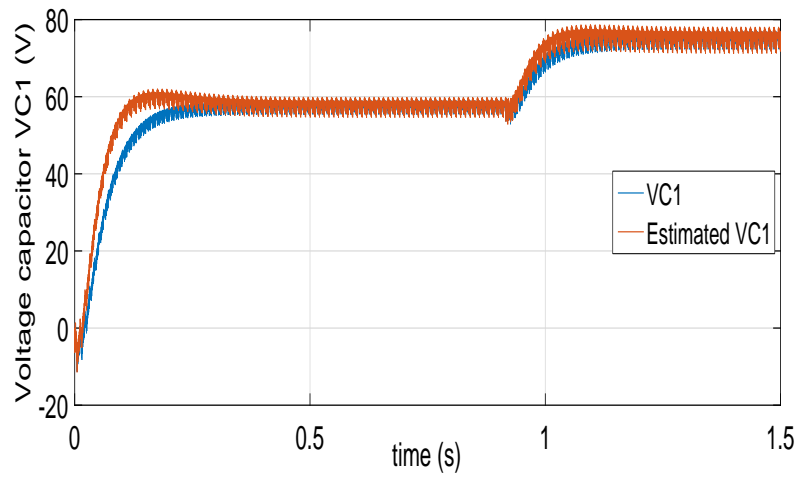

Fig. 10. Estimation of voltage capacitor $\mathrm{C} 1$

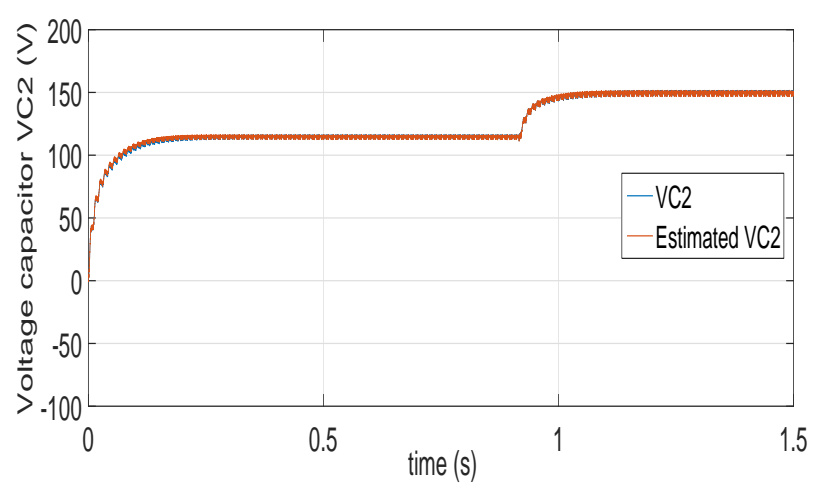

Fig. 11. Estimation of voltage capacitor $\mathrm{C} 2$

\section{B. Simulation Results}

The simulation results showing the performance of the minimal functional observer for the all voltages capacitor are presented in Fig. 10, 11, 12, 13, 14 and 15.

One can note that this observer keeps good properties in term of asymptotic convergence. In fact for $E=230 \mathrm{~V}$, the estimation of the voltage capacitors converge to its expected steady state values which are $V_{C 1}=57.5 \mathrm{~V}, V_{C 2}=115 \mathrm{~V}$ and $V_{C 3}=172.5 \mathrm{~V}$. In order to test the robustness of this observer, at $t=0.8 \mathrm{~s}$ the input voltage is set to $300 \mathrm{~V}$. The estimated

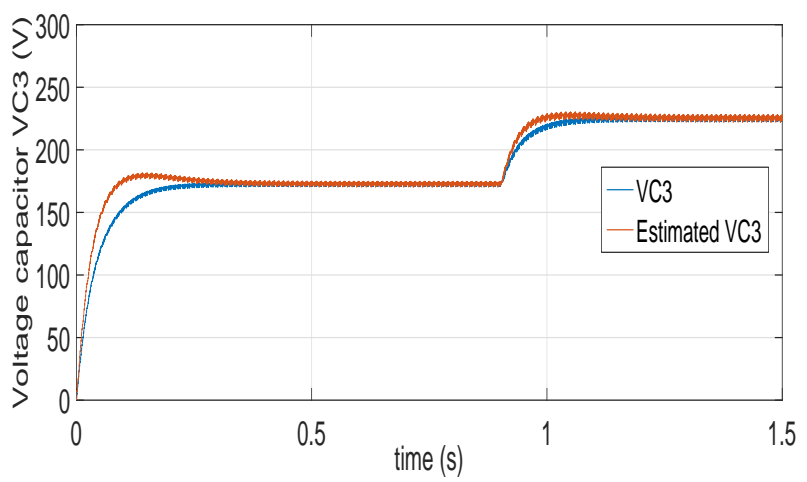

Fig. 12. Estimation of voltage capacitor $\mathrm{C} 3$

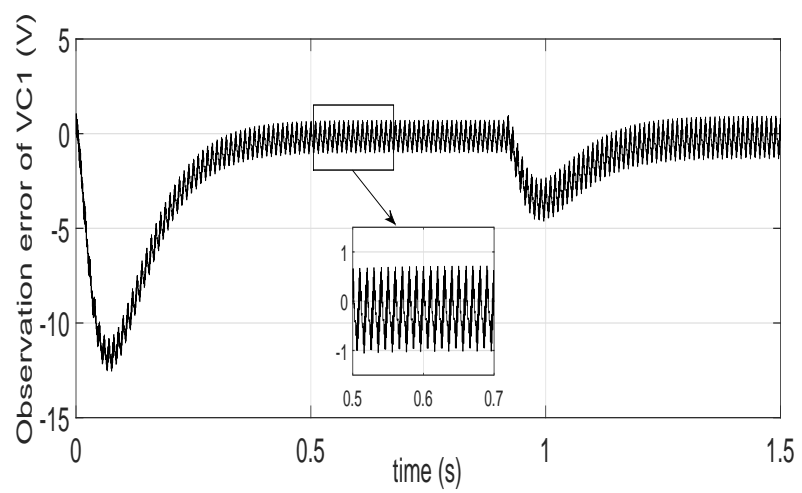

Fig. 13. Observation error of voltage capacitor $\mathrm{C} 1$

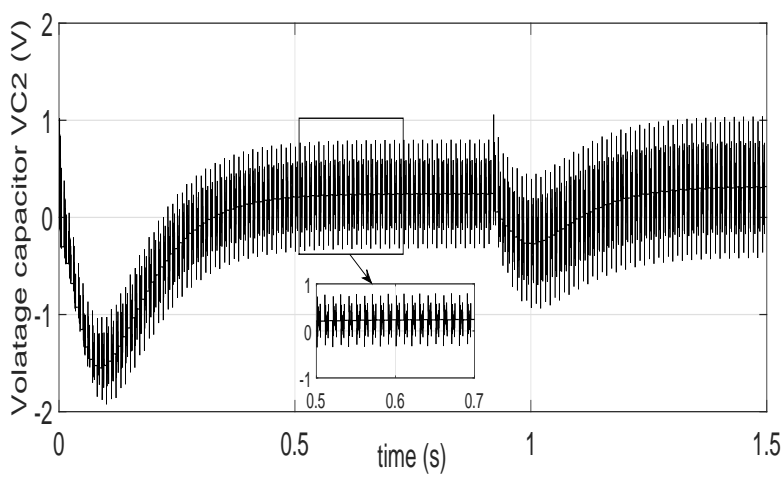

Fig. 14. Observation error of voltage capacitor C2

voltage values respond to the abrupt change of the input signal.

\section{Discussion}

As presented in Fig. 16, 17 and 18, the estimated capacitor voltage $V_{C j}$ is variable when the system switch to an observable mode, and keep constant when an other voltage is observable to avoid its re-observation. This result prove the respect of $Z(T N)$-observability criterion.

The estimation approach adopted in this paper are based on the $Z(T N)$-observability. The observer proposed in this paper

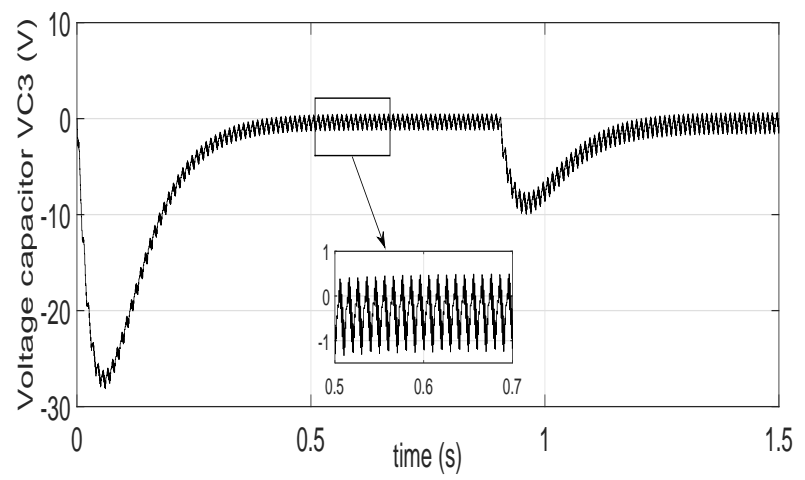

Fig. 15. Observation error of voltage capacitor C3 




Fig. 16. Zoom on voltage capacitor $\mathrm{C} 1$ and its estimation
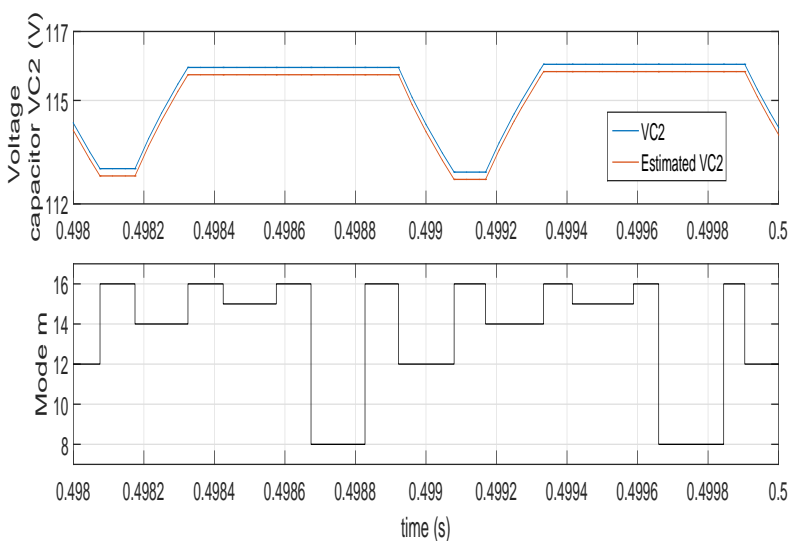

Fig. 17. Zoom on voltage capacitor $\mathrm{C} 2$ and its estimation

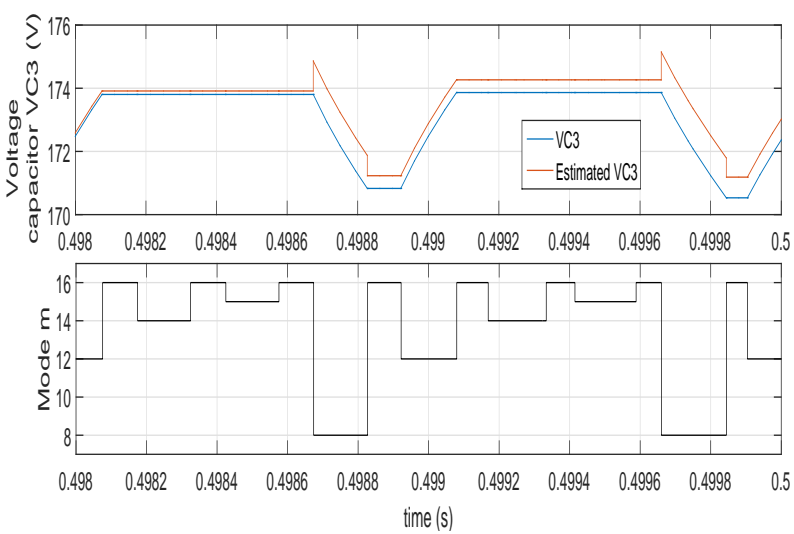

Fig. 18. Zoom on voltage capacitor C3 and its estimation is the minimal linear functional observer. On one hand this observer aims at reducing the system order and estimating each voltage separately on the other hand. A functional observer battery is designed for each voltage and for different operating modes. The adopted strategy in the functional observer design for each voltage capacitor is based on the classification of the operating modes which is a way to simplify the observation algorithm. In fact, When the system switch to the modes through it the voltage is not observable and constant, a zero dynamic is set. We deduce that the classification allows to specify the modes which intervene in the observation and neglects the other modes. As a results, the operating modes used for the voltage estimation are reduced from 16 to 8 modes (for four-cells converter). The simulation result prove that only the modes in which the voltage is observable, intervene on the observation dynamic.

This method becomes more complicated as the number of cells increases. In this case a huge number of battery of linear function observers are needed to estimate each voltages of the floating capacitors, there are also a significant number of modes.

\section{CONCLUSION}

In this paper the minimal single functional observer is proposed in order to estimate the capacitor voltages of the multicell converter which belongs to the hybrid dynamic system class. The problem of the capacitor voltages estimation is solved. Some simulations results of the four cells converters illustrate the performance and robustness of the proposed observer in the dynamic behaviour. This software sensor is an effective solution to detect the voltage imbalance instead of extra sensors. The deterioration of floating capacitors can be the cause of voltage unbalance. Consequently, our further work involves the application of the observer introduced in this paper in the presence of a capacitor default. Later, a synthesis of a closed-loop control using the estimated voltages in order to compensate this default is feasible .

\section{APPENDIX}

\section{A. Estimation of $V_{C 2}$}

To estimate the voltage capacitor $V_{C 2}$, the vector $P_{2}$ is set such as:

$$
P_{2}=\left[\begin{array}{llll}
0 & 1 & 0 & 0
\end{array}\right] \text {. }
$$

The observable mode of $V_{C 2}$ are modes $3,4,5,6,11,12$, 13,14 . The modes liable to the observation dynamic of the voltage $V_{C 2}$ are the modes 4 and 13. So, the observer gains are presented in Table V. $\lambda_{4}=1500$ and $\lambda_{13}=-1500$.

TABLE V. OBSERVER GAINS FOR MODES $3,4,5,6,11,12,13,14$

\begin{tabular}{|c|c|c|c|c|c|}
\hline $\mathrm{m}$ & $F_{m, 1}$ & $G_{m, 1}$ & $H_{m, 1}$ & $L_{m, 1}$ & $V_{m, 1}$ \\
\hline \hline 3 & 0 & 0 & -2500 & 1 & 0 \\
\hline 4 & -98.26 & 49.13 & -1.527 & 1 & 0.0983 \\
\hline 5 & 0 & 0 & 2500 & 1 & 0 \\
\hline 6 & 0 & 0 & 2500 & 1 & 0 \\
\hline 11 & 0 & 0 & -2500 & 1 & 0 \\
\hline 12 & 0 & 0 & -2500 & 1 & 0 \\
\hline 13 & -98.26 & 49.13 & 1527 & 1 & -0.0983 \\
\hline 14 & 0 & 0 & 2500 & 1 & 0 \\
\hline
\end{tabular}




\section{B. Estimation of $V_{C 3}$}

To estimate the voltage capacitor $V_{C 3}$, the vector $P_{3}$ is set such as:

$$
P_{3}=\left[\begin{array}{llll}
0 & 0 & 1 & 0
\end{array}\right] \text {. }
$$

Observable mode of $V_{C 3}$ are modes $5,6,7,8,9,10,11,12$. The modes liable to the observation dynamic of the voltage $V_{C 3}$ are the modes 8 and 9 . So, The observer gains are presented in Table VI.

TABLE VI. OBSERVER GAINS FOR MODES 5, 6, 7, 8, 9, 10, 11, 12

\begin{tabular}{|c|c|c|c|c|c|}
\hline $\mathrm{m}$ & $F_{m, 1}$ & $G_{m, 1}$ & $H_{m, 1}$ & $L_{m, 1}$ & $V_{m, 1}$ \\
\hline \hline 5 & 0 & 0 & -2500 & 1 & 0 \\
\hline 6 & 0 & 0 & -2500 & 1 & 0 \\
\hline 7 & 0 & 0 & -2500 & 1 & 0 \\
\hline 8 & -98.26 & 49.13 & -1527 & 1 & 0.0983 \\
\hline 9 & -98.26 & 49.13 & 1527 & 1 & -0.0983 \\
\hline 10 & 0 & 0 & 2500 & 1 & 0 \\
\hline 11 & 0 & 0 & 2500 & 1 & 0 \\
\hline 12 & 0 & 0 & 2500 & 1 & 0 \\
\hline
\end{tabular}

$\lambda_{8}=1500$ and $\lambda_{9}=-1500$.

\section{REFERENCES}

[1] M. Kasper, D. Bortis, and J. W. Kolar, "Scaling and balancing of multicell converters," in 2014 International Power Electronics Conference (IPEC-Hiroshima 2014-ECCE ASIA). IEEE, 2014, pp. 2079-2086.

[2] M. Jday and J. Haggège, "Modeling and neural networks based control of power converters associated with a wind turbine," in 2017 International Conference on Green Energy Conversion Systems (GECS). IEEE, 2017, pp. 1-7.

[3] A. K. Sadigh, V. Dargahi, and K. A. Corzine, "New active capacitor voltage balancing method for flying capacitor multicell converter based on logic-form-equations," IEEE Transactions on industrial electronics, vol. 64, no. 5, pp. 3467-3478, 2016.

[4] N. Gazzam and A. Benalia, "Observability analysis and observer design of multicellular converters," in 2016 8th International Conference on Modelling, Identification and Control (ICMIC). IEEE, 2016, pp. $763-$ 767.

[5] J. Van Gorp, M. Defoort, M. Djemai, and K. C. Veluvolu, "Fault detection based on higher-order sliding mode observer for a class of switched linear systems," IET Control Theory \& Applications, vol. 9, no. 15 , pp. 2249-2256, 2015.

[6] K. Benmansour, J. De Leon, and M. Djemai, "Adaptive observer for multi-cell chopper," in Second International Symposium on Cоттиnications, Control and Signal Processing ISCCSP, Marrakech, Maroc, 2006.

[7] M. Ghanes, F. Bejarano, and J.-P. Barbot, "On sliding mode and adaptive observers design for multicell converter," in 2009 American Control Conference. IEEE, 2009, pp. 2134-2139.
[8] M. Jday, P.-E. Vidal, J. Haggège, and F. Rotella, "Observability and sliding mode observer design for multi-cell series converter," in 2019 6th International Conference on Control, Decision and Information Technologies (CoDIT). IEEE, 2019, pp. 1486-1491.

[9] J. Lin, Y. Shi, Z. Gao, and J. Ding, "Functional observer for switched discrete-time singular systems with time delays and unknown inputs," IET Control Theory \& Applications, vol. 9, no. 14, pp. 2146-2156, 2015.

[10] I. Sakhraoui, B. Trajin, and F. Rotella, "Application of linear functional observers for the thermal estimation in power modules," 2018.

[11] K. Berkoune, P.-E. Vidal, and F. Rotella, "Modélisation générique pour les stratégies de modulation des onduleurs multiniveaux: application aux onduleurs à capacités flottantes," 2016.

[12] J. Rodriguez, J.-S. Lai, and F. Z. Peng, "Multilevel inverters: a survey of topologies, controls, and applications," IEEE Transactions on industrial electronics, vol. 49, no. 4, pp. 724-738, 2002.

[13] A. Bouarfa, M. Bodson, and M. Fadel, "A fast active-balancing method for the 3-phase multilevel flying capacitor inverter derived from control allocation theory," IFAC-PapersOnLine, vol. 50, no. 1, pp. 2113-2118, 2017.

[14] W. Kang and J.-P. Barbot, "Discussions on observability and invertibility," IFAC Proceedings Volumes, vol. 40, no. 12, pp. 426-431, 2007.

[15] B. Amghar, A. Darcherif, and J.-P. Barbot, " $Z$ (tn)-observability and control of parallel multicell chopper using petri nets," IET Power Electronics, vol. 6, no. 4, pp. 710-720, 2013.

[16] A. Smati, W. Chagra, and M. Kssouri, "Fault-tolerant model predictive control for az (tn)-observable linear switching systems," International Journal Of Advanced Computer Science And Applications, vol. 8, no. 6, pp. 365-374, 2017.

[17] F. J. Bejarano, M. Ghanes, and J.-P. Barbot, "Observability and observer design for hybrid multicell choppers," International Journal of Control, vol. 83, no. 3, pp. 617-632, 2010.

[18] J. Van Gorp, M. Defoort, M. Djemai, and N. Manamanni, "Hybrid observer for the multicellular converter," IFAC Proceedings Volumes, vol. 45, no. 9, pp. 259-264, 2012.

[19] M. Darouach, "Existence and design of functional observers for linear systems," IEEE Transactions on Automatic Control, vol. 45, no. 5, pp. 940-943, 2000.

[20] H. Trinh, T. Tran, and S. Nahavandi, "Design of scalar functional observers of order less than $(\nu$ - 1)," International journal of control, vol. 79, no. 12, pp. 1654-1659, 2006.

[21] H. Trinh and T. Fernando, "On the existence and design of functional observers for linear systems," in 2007 International Conference on Mechatronics and Automation. IEEE, 2007, pp. 1974-1979.

[22] T. L. Fernando, H. M. Trinh, and L. Jennings, "Functional observability and the design of minimum order linear functional observers," IEEE Transactions on Automatic Control, vol. 55, no. 5, pp. 1268-1273, 2010.

[23] F. Rotella and I. Zambettakis, "A note on functional observability," IEEE Transactions on Automatic Control, vol. 61, no. 10, pp. 3197-3202, 2015.

[24] A. Ben-Israel and T. N. Greville, Generalized inverses: theory and applications. Springer Science \& Business Media, 2003, vol. 15. 